\title{
An Approach to Modeling and Scaling of Hysteresis in Magnetic Materials. Magnetization Curve
}

\author{
K.Z. SOKALSKI* \\ Institute of Computer Science, Częstochowa University of Technology, \\ al. Armii Krajowej 17, 42-200 Częstochowa, Poland \\ (Received January 8, 2015; in final form February 5, 2015)
}

\begin{abstract}
A new mathematical model of hysteresis loop has been derived. Model consists in an extension of $\tanh (\cdot)$ by extending the base of exp function into an arbitrary positive number. The presented model is self-similar and invariant with respect to scaling. Scaling of magnetic hysteresis loop has been done using the notion of homogeneous function in general sense.
\end{abstract}

DOI: 10.12693/APhysPolA.127.850

PACS: 75.50.-y, 75.60.-d, 89.75.Da

\section{Introduction}

In the two last decades the following models have been accepted for a proper description of the hysteresis loop:

- Jiles-Atherton [1, 2] and Szczygłowski's supplement [3] as well as Chwastek's modification [4],

- Harrison $[5,6]$,

- Takács [7, 8],

- Zirka-Moroz [9].

For older approaches to the problem of magnetization hysteresis we refer to books by Bertotti [10] and Cullity [11]. For more recent approaches we refer to the monograph [12]. Very recent paper by Zirka et al. [13] is also remarkable due to new approach. Despite the multiplicity of hysteresis models developed to date, there is no model in which self-similarity can be expressed by homogeneous functions in general sense. It is necessary to remind that a far more general concept than universality is scaling which applies very fruitfully to power losses in magnetic materials [14]. Since these two problems are related by the area of hysteresis loop, there should exist an analogical representation of scaling for the magnetization processes. The goal of the present paper is to create a mathematical model which describes hysteresis and enables to express its self-similarity by the homogeneous function in general sense. This function should enable us to reproduce all magnetization processes inside a major loop which will play a role of an envelope for these processes.

It is well known that $\tanh (\cdot)$ suits for model of initial magnetization function. It describes properly the saturation for both asymptotic values of the magnetic field: $H \rightarrow \pm \infty$ as well as the behavior of magnetization in

*e-mail: ksokalski76@gmail.com the neighborhood of origin. The mentioned above paper [7] starts from tanh(.) and describes phenomenological model which gives complete description of the magnetic hysteresis. Moreover, this mapping helps to solve problems of modeling the core losses in presence of DC bias by the projection of the magnetic field just onto the initial curve [15]. In this paper we present mathematical model of hysteresis in soft magnetic materials which bases on an extension of the function $\tanh (\cdot)$. The goal of this paper is to derive mathematical model which properly describes the magnetization hysteresis and it enables us to perform scaling of the hysteresis loop basing on notions of self-similar system and homogeneous function in general sense $[14,16]$.

This paper is organized as follows. In Sect. 1 we introduce the extended $\tanh (\cdot)$ function from which we derive its basic properties. In Sect. 2 we deal with self-similarity of the considered model and scaling. Conclusions are presented in Sect. 3.

\section{Extension of $\tanh (\cdot)$ function and mathematical model of hysteresis}

Let us start from the definition of $\tanh (\cdot)$ :

$$
\tanh (x)=\frac{\mathrm{e}^{x}-\mathrm{e}^{-x}}{\mathrm{e}^{x}+\mathrm{e}^{-x}}
$$

where e is the base of the so-called natural logarithm. So, let us generalize (1) by introducing the four bases instead of e:

$$
\operatorname{tanH}(a, b, c, d \mid x)=\frac{a^{x}-b^{-x}}{c^{x}+d^{-x}},
$$

where $a, b, c, d$ are arbitrary positive numbers.

\subsection{Hysteresis in (magnetic field, magnetization) plane}

In the first step we will derive a model of major hysteresis loop which is an envelope of the whole family of loops of the particular considered case. Let us write down the model expression for initial magnetization curve

$$
M_{P}(X)=M_{0} P(X, \epsilon) ; \quad X \in\left[0, X_{\max }\right],
$$

where $M_{0}$ is magnetization corresponding to saturation expressed in T: [T], $X \in\left\{0, X_{\max }\right\}, X=\frac{H}{h}$ where $H$ is 
magnetic field, $h$ is a parameter of the magnetic field dimension $\left[\mathrm{A} \mathrm{m}^{-1}\right]$ to be determined and where function $P(X, \epsilon)$ is of the following form:

$$
P(X, \epsilon)=\frac{a^{X-\epsilon}-b^{-X+\epsilon}}{c^{X-\epsilon}+d^{-X+\epsilon}},
$$

where $\epsilon$ is modeling parameter of the order of $\theta / 2$. Let the upper branch of the hysteresis loop and the lower one are of the following forms:

$$
M_{F}(X)=M_{0} F(X, \theta) ; \quad M_{G}(X)=M_{0} G(X, \theta),
$$
where

$$
\begin{aligned}
& F(X, \theta)=\frac{a^{X+\theta}-b^{-X-\theta}}{c^{X+\theta}+d^{-X-\theta}}, \\
& G(X, \theta)=\frac{a^{X-\theta}-b^{-X+\theta}}{c^{X-\theta}+d^{-X+\theta}} .
\end{aligned}
$$

$\theta$ is a model parameter depending on $X_{\max }$.

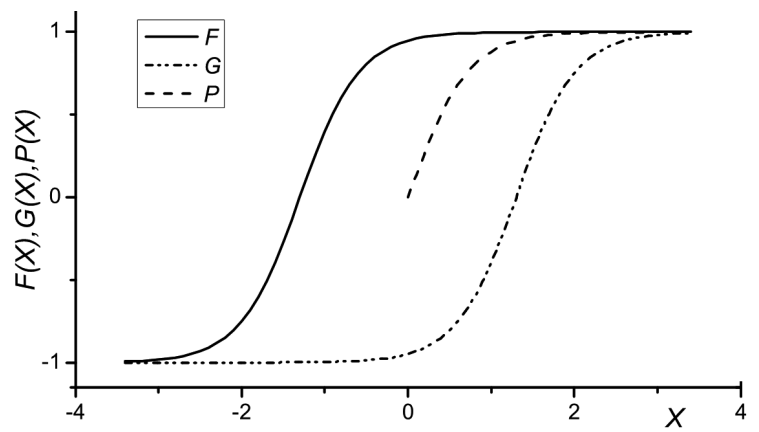

Fig. 1. A model of nucleation type hysteresis constructed with functions $F, P$ and $G$ according to (6), (7), and (4), respectively.

Let us consider for illustration the following example: $M_{0}=1, a=4, b=4, c=4, d=4, \theta_{i}=1.3$ for $i=a, b, c, d$. The magnetization as a function of the magnetic field possesses horizontal asymptotes for $H \rightarrow \pm \infty$. This means for our modeling that the functions $F(X, \theta)$ and $G(X, \theta)$ have to possess the same asymptotic properties. These components get equal values for $|H|=\infty$. In fact due to the uncertainty of measured magnitudes it is possible to accept the saturation points at $X=X_{\min }$ and $X=X_{\max }$ being the end points of the hysteresis loop. Therefore, the modeling process has to ensure that the initial function $P(H)$ matches the origin and the upper end point of the loop. This effect can be controlled by the $\theta$ parameter, see Fig. 1 . Values of $X_{\max }$ are determined by the following relation:

$$
\left|F\left(X_{\max }, \theta\right)-G\left(X_{\max }, \theta\right)\right| \leq|\psi|,
$$

where $\psi$ is measure of uncertainty of $\left|M_{F}(X)-M_{G}(X)\right|$ in dimensionless units: $\psi=\sup _{X}\left|M_{F}(X)-M_{G}(X)\right| / M_{0}$.

\subsection{Scaling of magnetization's hysteresis loop}

We present the scaling procedure on example of the both branches of a major loop. Functions which have to be scaled (5)-(7) consist of exponential functions and their arguments are exponents. In such a case the scaling can be performed on the basis of these functions, while the exponents can be converted by gauge transformations. Let us assume that there exist real numbers $(\alpha, \beta, \gamma, \delta, \nu) \in \boldsymbol{R}^{5}$ such that $\forall \lambda \in \boldsymbol{R}_{+}$and $\forall \chi \in \boldsymbol{R}$ the following relations hold:

$$
\begin{aligned}
& \frac{M_{F}(X)}{M_{0}} \lambda^{\nu}=\frac{\left(\lambda^{\alpha} a\right)^{X+\theta} a^{\chi}-\left(\lambda^{\beta} b\right)^{-X-\theta} b^{-\chi}}{\left(\lambda^{\gamma} c\right)^{X+\theta} c^{\chi}+\left(\lambda^{\delta} d\right)^{-X-\theta} d^{-\chi}}, \\
& \frac{M_{G}(X)}{M_{0}} \lambda^{\nu}=\frac{\left(\lambda^{\alpha} a\right)^{X-\theta} a^{\chi}-\left(\lambda^{\beta} b\right)^{-X+\theta} b^{-\chi}}{\left(\lambda^{\gamma} c\right)^{X-\theta} c^{\chi}+\left(\lambda^{\delta} d\right)^{-X+\theta} d^{-\chi}},
\end{aligned}
$$

then $M_{F}(X)$ and $M_{G}(X)$ make a set of homogeneous functions in general sense.

\subsection{Group symmetry of the considered model}

$\alpha, \beta, \gamma, \delta, \nu$ are scaling exponents and $\chi$ is a gauge transformation parameter. We have assumed that the gauge transformation acts directly on the argument of the magnetization function: $M_{F}(X) \rightarrow M_{F}(X+\chi)$, while scaling acts directly on each base of exponential functions, for example: $a^{X} \rightarrow\left(\lambda^{\alpha} a\right)^{X}$. We will show that this action can be transformed to an operator acting directly on the linear function of $X$. The derived transformation (8) depends on two parameters: $\lambda$ and $\chi$. Each of them generates one parameter group: multiplicative group $\mathcal{G}_{\lambda}$ and additive one $\mathcal{G}_{\chi}$, respectively. By the definitions how do the scaling and gauge transformations act on the model equations the group of symmetry of (8) is the following semidirect product [17]:

$$
\mathcal{G}_{\lambda, \chi}=\mathcal{G}_{\lambda} \rtimes \mathcal{G}_{\chi} .
$$

Group action in $\mathcal{G}_{\lambda, \chi}$ is defined as follows. Let $\left\{p_{1}, \chi_{1}\right\} \in$ $\mathcal{G}_{\lambda, \chi}$ and $\left\{p_{2}, \chi_{2}\right\} \in \mathcal{G}_{\lambda, \chi}$. Then

$$
\left\{p_{2}, \chi_{2}\right\} \rtimes\left\{p_{1}, \chi_{1}\right\}=\left\{p_{2} p_{1}, p_{2} \chi_{1}+\chi_{2}\right\} .
$$

The group structure of $\mathcal{G}_{\lambda, \chi}$ will play important role in derivation relations between hysteresis loops.

\subsection{Symmetric model}

Now we are ready to prove that the symmetric model of the hysteresis loop is invariant with respect to $\lambda_{i}, \chi_{j} \in \mathcal{G}_{\lambda, \chi}$. By the symmetric model we mean a case characterized by the following relations between basis of (4), (6), (7):

$$
a=b=c=d \text {. }
$$

Otherwise a model is non-symmetric. For further considerations we take into account the following formula for $M_{F}(X), M_{G}(X), M_{P}(X)$ :

$$
\begin{aligned}
& \frac{M_{F}(X)}{M_{0}}=\frac{(a)^{X+\theta}-(a)^{-X-\theta}}{(a)^{X+\theta}+(a)^{-X-\theta}}, \\
& \frac{M_{G}(X)}{M_{0}}=\frac{(a)^{X-\theta}-(a)^{-X+\theta}}{(a)^{X-\theta}+(a)^{-X+\theta}}, \\
& \frac{M_{P}(X)}{M_{0}}=\frac{(a)^{X+\epsilon}-(a)^{-X-\epsilon}}{(a)^{X+\epsilon}+(a)^{-X-\epsilon}}
\end{aligned}
$$

Theorem: If basis of the model (4), (6) and (7) satisfy (11) then the model of hysteresis loop is invariant with respect to scaling and gauge transformation. 
Proof: Let us assume an action of a group element belonging to $\mathcal{G}_{\lambda, \chi}$. For the symmetry condition (11) the relation (8) takes the following form:

$$
\begin{aligned}
& \frac{M_{F}(X)}{M_{0}} \lambda^{\nu}=\frac{\left(\lambda^{\alpha} a\right)^{X+\theta} a^{\chi}-\left(\lambda^{\alpha} a\right)^{-X-\theta} a^{-\chi}}{\left(\lambda^{\alpha} a\right)^{X+\theta} a \chi+\left(\lambda^{\alpha} a\right)^{-X-\theta} a^{-\chi}}, \\
& \frac{M_{G}(X)}{M_{0}} \lambda^{\nu}=\frac{\left(\lambda^{\alpha} a\right)^{X-\theta} a^{\chi}-\left(\lambda^{\alpha} a\right)^{-X+\theta} a^{-\chi}}{\left(\lambda^{\alpha} a\right)^{X-\theta} a^{\chi}+\left(\lambda^{\alpha} a\right)^{-X+\theta} a^{-\chi}},
\end{aligned}
$$

where we have to remember that according to (10) the order of action is relevant. First the scaling has to be done before the gauge transformation. According to the assumptions just above (8) we are free to assume that

$$
\lambda^{\alpha}=a^{p-1} \text {. }
$$

Then the relations (15) after simple evaluations take the following form:

$$
\begin{aligned}
\frac{M_{F}(X)}{M_{0}} a^{n} & =\frac{(a)^{p(X+\theta)+\chi}-(a)^{p(-X-\theta)-\chi}}{(a)^{p(X+\theta)+\chi}+(a)^{p(-X-\theta)-\chi}}, \\
\frac{M_{G}(X)}{M_{0}} a^{n} & =\frac{(a)^{p(X-\theta)+\chi}-(a)^{p(-X+\theta)-\chi}}{(a)^{p(X-\theta)+\chi}+(a)^{p(-X+\theta)-\chi}},
\end{aligned}
$$

where $n=\frac{\nu}{\alpha}(p-1)$ and $p \in \boldsymbol{R} \backslash\{0\}$. Introducing the following new variables:

$$
M_{0}^{\prime}=M_{0} a^{-n}, \quad X^{\prime}=p X+\chi, \quad \theta^{\prime}=p \theta,
$$

we get (12). The initial magnetization curve (14) is invariant with respect to the scaling and the gauge transformations as well. The proof goes the same way as for (12) and (13).

\subsection{Interpretation of scaling and gauge transformation}

In order to make an interpretation of the derived scaling and gauge transformation we present results of their action on the hysteresis models, see Figs. 2-4. Figure 2 presents how pure gauge transformations generate a displacement of transformed loops along the horizontal axis. Figure 3 presents compression of a loop along the vertical axis under the scaling. Finally, for large value of the scaling parameter $p$ the transformed loops resemble the Preisach hysterons (Fig. 4 [18]).

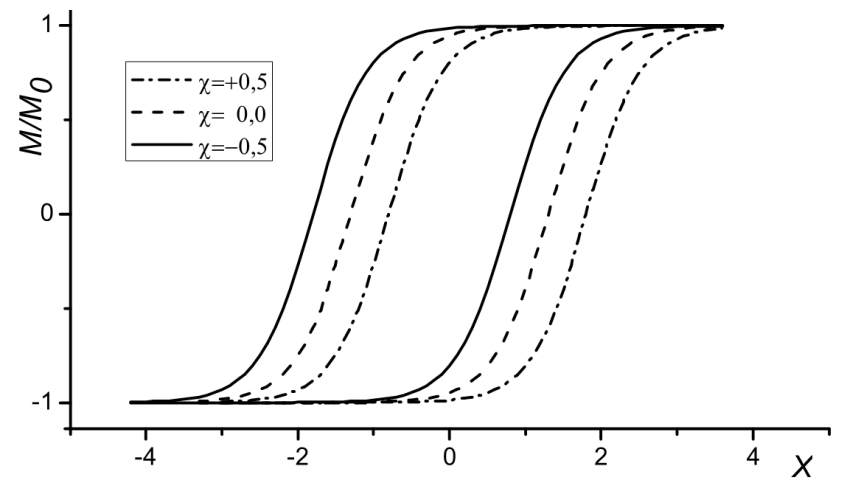

Fig. 2. Magnetic hysteresis family for $p=1, n=1$, $\theta=1.3, \nu / \alpha=1$.

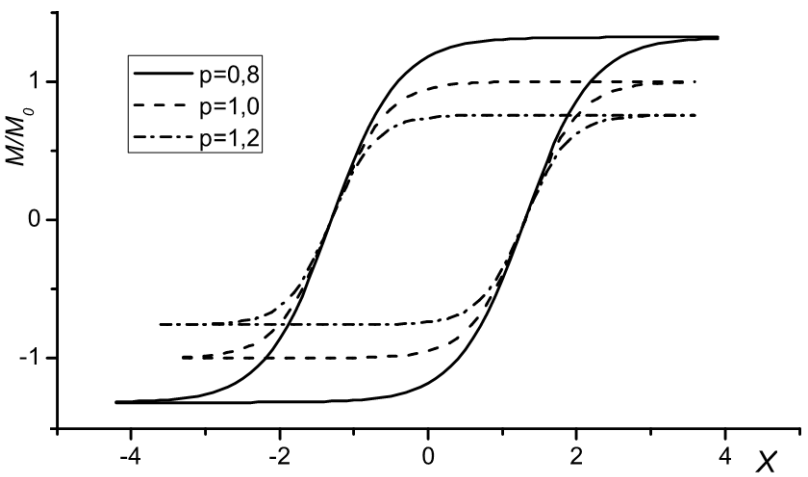

Fig. 3. Magnetic hysteresis family for $\chi=0, n=1$, $\theta=1.3, \nu / \alpha=1$.

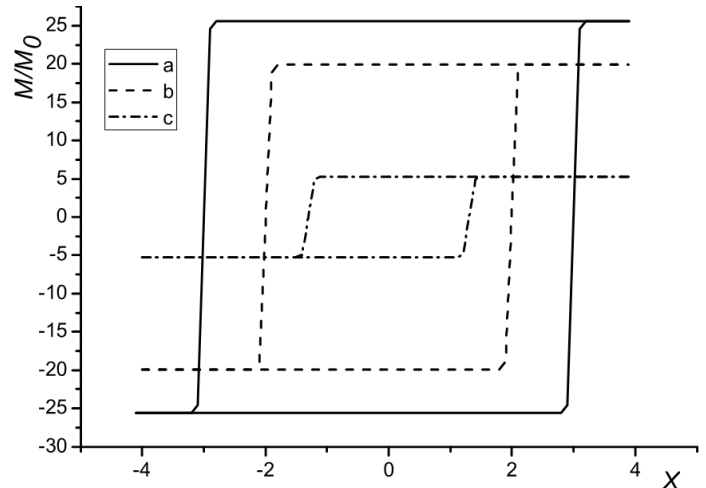

Fig. 4. Magnetic hysteresis family for the following values of the model and scaling parameters: (a) $\theta=3$, $p=14, \nu / \alpha=-0.18, \chi=0$, (b) $\theta=2, p=13$, $\nu / \alpha=-0.18, \chi=0$, (c) $\theta=1.3, p=13, \nu / \alpha=0$, $\chi=0$.

\subsection{Division of the hysteresis loops space in $\mathcal{G}_{\lambda, \chi}$}

Let $\boldsymbol{L}$ be the space of hysteresis loops created according to algorithm described in Sect. 2.4 for all the values $a>1$. Let us consider the two loops $\boldsymbol{l}_{i} \in \boldsymbol{L}$ and $\boldsymbol{l}_{j} \in \boldsymbol{L}$. By definition, $\boldsymbol{l}_{i}$ and $\boldsymbol{l}_{j}$ belong to the same class if there exists $\left\{p_{j}, \chi_{j}\right\} \in \mathcal{G}_{\lambda, \chi}$ such that

$$
\boldsymbol{l}_{j}=\left\{p_{j}, \chi_{j}\right\} \boldsymbol{l}_{i},
$$

where the action of $\left\{p_{j}, \chi_{j}\right\}$ in the $\boldsymbol{L}$ space is defined by (15)-(18). Subset $\boldsymbol{L}_{i}$ consisting of the all $\boldsymbol{l}_{j}$ satisfying (19), constitutes the class $\boldsymbol{l}_{i}$ so-called orbit. Equation (19) is equivalence relation due to the group structure of $\boldsymbol{G}_{\lambda, \chi}$. Therefore, (19) constitutes division of $\boldsymbol{L}$. Any two loops of the same base $a$ are equivalent due to the structure of $\mathcal{G}_{\lambda, \chi}$ and the two loops having different basis belong to two different orbits. All orbits cover the full $\boldsymbol{L}$ space.

\section{Conclusions}

By physical models of the hysteresis loops we try to explain origin of the magnetic materials properties. Whereas in designing the magnetic materials we need 
analytic formulae returning real values of physical magnitudes which are relevant in this process. Just the mathematical models together with an experimental data constitute powerful tool for designers $[19,20]$. In this paper we have derived the simple model which is promising for the applications mentioned above. The introduced group theoretical methods in analysis of the hysteresis loops help us to divide the hysteresis loops space into orbits in $\mathcal{G}_{\lambda, \chi}$. This division enables us to create a minimal set of independent loops which constitutes a base in the hysteresis loops space.

There are needs to extend the described model onto induction. Work on this subject is in progress and concentrates on the three following problems: modeling the proper asymptotic features when $H \rightarrow \pm \infty$, investigating the algebraic structure generated by (6), (8), and deriving subsets of singular parameters values.

\section{References}

[1] D.C. Jiles, D.L. Atherton, IEEE Trans. Magn. 19, 2183 (1983).

[2] D.C. Jiles, D.L. Atherton, J. Appl. Phys. 55, 2115 (1984).

[3] J. Szczygłowski, J. Magn. Magn. Mater. 223, 97 (2001).

[4] K. Chwastek, Physica B 403, 2484 (2008).

[5] R.G. Harrison, IEEE Trans. Magn. 39, 950 (2003).

[6] R.G. Harrison, IEEE Trans. Magn. 45, 1922 (2009).

[7] J. Takács, COMPEL: Int. J. Comput. Math. Electr. Electron. Eng. 20, 1014 (2001).
[8] J. Takács, Mathematics of Hysteretic Phenomena, Wiley-VCH, Weinheim 2003

[9] S.E. Zirka, Y.I. Moroz, IEEE Trans. Magn. 35, 2090 (1999).

[10] G. Bertotti, Hysteresis in Magnetism, Academic Press, San Diego 1998.

[11] B.D. Cullity, Introduction to Magnetic Materials, Addison-Wesley, Reading, Massachusetts 1972.

[12] The Science of Hysteresis, Vols. 1-3, Eds. G. Bertotti, I.D. Mayergoyz, Academic Press, Amsterdam 2005.

[13] S.E. Zirka, Y.I. Moroz, R.G. Harrison, N. Chiesa, IEEE Trans. Power Deliv. 29, 552 (2014).

[14] K. Sokalski, J. Szczygłowski, M. Najgebauer, W. Wilczy nski, COMPEL: Int. J. Comput. Math. Electr. Electron. Eng. 26, 640 (2007).

[15] A. Ruszczyk, K. Sokalski, J. Szczygłowski, arXiv:1309.0022.

[16] G.I. Barenblatt, Scaling, Cambridge Texts in Applied Mathematics, Cambridge University Press, Cambridge 2003.

[17] M. Hamermesh, Group Theory and Its Application to Physical Problems, Pergamon Press, London 1962.

[18] F. Preisach, Z. Phys. 94, 277 (1935).

[19] B Ślusarek, B. Jankowski, K. Sokalski, J. Szczygłowski, J. Alloys. Comp. 581, 699 (2013).

[20] K.Z. Sokalski, B. Jankowski, B. Ślusarek, Materials Sciences and Applications 5, 1040 (2014). 